\title{
COMPARISON OF SINGLE AND MULTI-SCALE METHOD FOR LEAF AND WOOD POINTS CLASSIFICATION FROM TERRESTRIAL LASER SCANNING DATA
}

\author{
Hongqiang Wei ${ }^{1}$, Guiyun Zhou ${ }^{1} *$, Junjie Zhou ${ }^{1}$ \\ ${ }^{1}$ School of Resources and Environment, University of Electronic Science and Technology of China, Chengdu, Sichuan 611731, China \\ - (hongqiang.wei, guiyun.zhou, junjie.zhou) zhouguiyun@uestc.edu.cn
}

KEY WORDS: Scale, Leaf and wood classification, Terrestrial Laser Scanning, Tree point cloud, Machine Learning

\begin{abstract}
:
The classification of leaf and wood points is an essential preprocessing step for extracting inventory measurements and canopy characterization of trees from the terrestrial laser scanning (TLS) data. The geometry-based approach is one of the widely used classification method. In the geometry-based method, it is common practice to extract salient features at one single scale before the features are used for classification. It remains unclear how different scale(s) used affect the classification accuracy and efficiency. To assess the scale effect on the classification accuracy and efficiency, we extracted the single-scale and multi-scale salient features from the point clouds of two oak trees of different sizes and conducted the classification on leaf and wood. Our experimental results show that the balanced accuracy of the multi-scale method is higher than the average balanced accuracy of the single-scale method by about $10 \%$ for both trees. The average speed-up ratio of single scale classifiers over multi-scale classifier for each tree is higher than 30 .
\end{abstract}

\section{INTRODUCTION}

Terrestrial laser scanning (TLS) provides a revolutionary way of quantifying individual tree characteristics, with details and accuracy that satellite laser scanning (SLS) and airborne laser scanning (ALS) cannot match (Tao et al., 2015). Significant progress has been made using TLS data to calculate diameter at breast height $(\mathrm{DBH})$, leaf area index (LAI), plant biomass, virtual projections, gap fraction, etc. (Dassot et al., 2011). The inventory measurements and canopy characterization of trees help ecologists and botanists build more accurate models for large amount of fine-scale research.

The retrieval of many parameters of tree from TLS data requires the classification of leaf and wood points to improve accuracy and reduce complexities. The classification of tree point clouds into leaf and wood points is a challenge. According to Wang et al. (2017), two types of methods have emerged for this problem.
The first-type method uses intensity information of returned laser pulse (Pfeifer et al., 2007; Pfennigbauer and Ullrich, 2010; Béland et al., 2014). The assumption of this approach is that there are significant differences among the optical properties of different components of a tree at the operating wavelength of the laser system (Tao et al., 2015; Wang et al., 2017). Trees of different species may respond similarly to the laser wavelength , meaning that the intensity-based approach cannot be used for some tree species. In addition, the intensity values need an instrument specific radiometric calibration before it can be used for leaf and wood classification (Pfennigbauer and Ullrich, 2010; Calders et al., 2017). According to Hakala et al. (2012), multiwavelength scanners have huge potential for improved accuracy and efficiency in comparison with traditional monochromatic laser scanners. However, this type of scanners are still in an early development stage and are not available from commercial manufacturers. The second-type method is referred to as the geometry-based method, which uses three dimensional

* Corresponding author 
coordinates of objects captured by a laser scanner (Tao et al., 2015; Wang et al., 2017). Tao et al. (2015) proposed a geometrybased method that focuses on leaf and wood classification of TLS data. Their method extracts the skeleton of trees and then separates the leaf and wood points. Ma et al. (2016) developed a method based on the Lalonde's framework that used the spatial distribution patterns of the manually selected training points of each class to drive the Gaussian mixture model (GMM) for leaf and wood classification (Lalonde et al., 2006). Yun et al. (2016) presented another method based on Dey's method, which calculated the shape, normal vector distribution, structure tensor of tree point clouds and used the semi-supervised support vector machine (SVM) classifier to separate leaf and wood points (Dey et al., 2012). Recently, Li et al. (2017) proposed the normal difference method based on the differences in the structures of leaf and non-leaf components of trees. To find better machine learning classifiers and salient features, Wang et al. (2017) examined four geometry-based machine learning classifiers and many salient features that were widely adopted in other classification tasks (Brodu et al., 2012; Weinmann et al., 2013, 2015, 2017) and found that machine learning classifiers and several salient features could efficiently separate leaf and wood points from TLS data with high accuracy.

In Wang's experiments, he only uses single-scale salient features to training classifiers. However, the surfaces of trees are heterogeneous and their distinctive properties are seldom defined at one specific scale. Therefore, using multi-scale salient features in this problem holds huge potential for improved accuracy (Brodu et al., 2012). The lack of comparative studies on the abilities of single-scale and multi-scale salient features to characterize the spatial patterns calls for further research on how single-scale and multi-scale salient features affect the classification accuracy and efficiency.

In this study, we examine the accuracy and efficiency of singleand multi-scale leaf and wood classification methods to find better classification strategy based on the Brodu's framework.

\section{MATERIALS}

The study site is located at the Jigong Mountain National Nature Reserve (114 $02^{\prime}$ E, $\left.31^{\circ} 50^{\prime} \mathrm{N}\right)$, Henan Province, China. The TLS data of two oak trees of different sizes were acquired from four scan positions by Leica ScanStation P40 in April, 2016. The Registration and Edit module of Leica Cyclone 9.1.4 software are used to preprocess tree point cloud. The acquired point cloud was further manually cleaned, as the point cloud of other trees and ground should be removed. The cleaned point cloud for tree 1 and tree 2 contains 3,065,470 and 11,373,009 points, respectively. The average distance between two adjacent points is $\sim 3 \mathrm{~mm}$. To assess the classification accuracy of single- and multi-scale methods, we manually identified leaf and wood points of the two trees. For tree 1, 1,948,299 and 1,117,171 points belong to leaf and wood, respectively. For tree 2, $6,799,597$ points belong to leaf and 4,573,412 points to wood. The original data of tree 1 and tree 2 are shown in Figure 1(a) and Figure 2(a), respectively. 


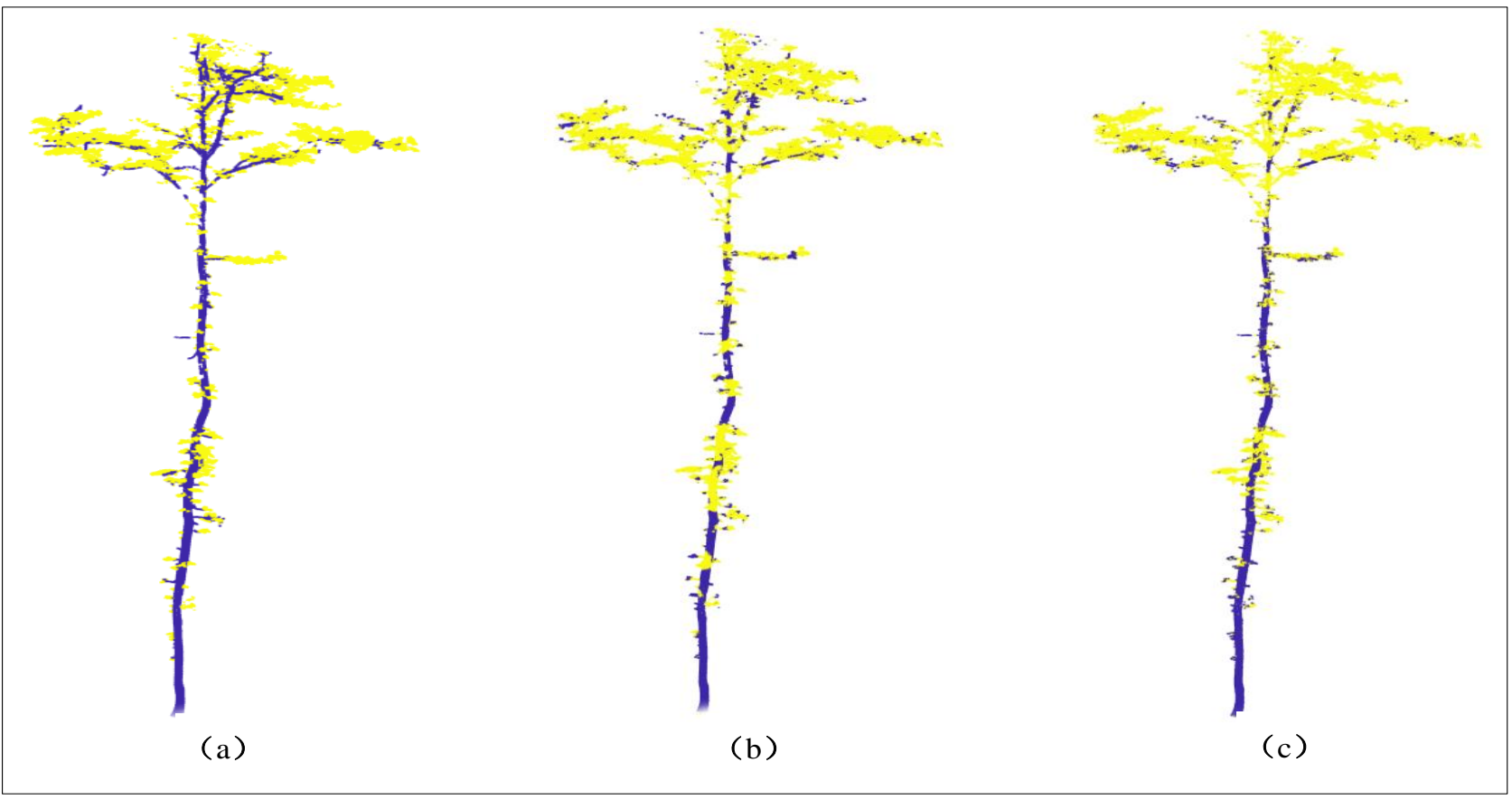

Figure 1. The point cloud of tree 1. (a) the manually classified point cloud; (b) the classification result of single-scale method ( $r=$ $0.3 \mathrm{~m})$; (c) the classification result of multi-scale method $(r=0.02 \mathrm{~m}-1 \mathrm{~m})$.

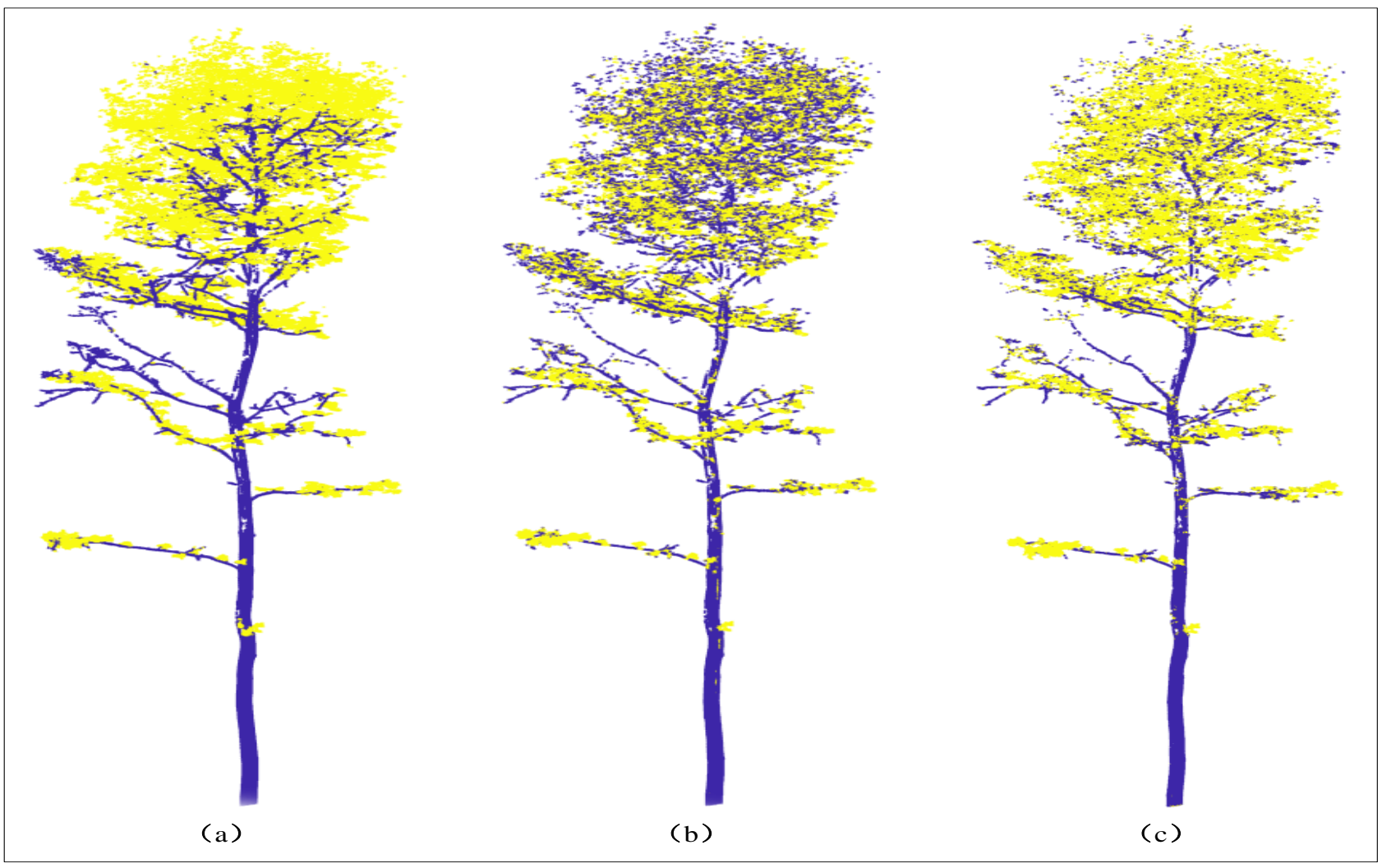

Figure 2. The point cloud of tree 2. (a) the manually classified point cloud; (b) the classification result of single-scale method $(r=$ $0.14 \mathrm{~m})$; (c) the classification result of multi-scale method $(r=0.02 \mathrm{~m}-1 \mathrm{~m})$. 


\section{METHODS}

\subsection{Feature Calculation}

Let $q=(x, y, z) \in R^{3}$ be a point in the three dimensional space. $Q$ $=\left\{q_{i} \in R^{3} \mid i=1, \ldots, N\right\}$ denotes the tree point cloud. The scale $r$ in our paper is defined as the radius of the ball centered on a point of interest. For each point in $Q$, the neighborhood ball is computed at one or more given scale(s). The Principal Component Analysis (PCA) is applied to the points in that ball (Shaw, 2013).

The ordered eigenvalues resulting from the PCA for point $q_{i}$ are $\lambda_{1} \geq \lambda_{2} \geq \lambda_{3}$, which is used to infer the local spatial distribution pattern of this point. Let $P_{i}=\lambda_{i} /\left(\lambda_{1}+\lambda_{2}+\lambda_{3}\right)$. If $P_{1} \gg P_{2} \approx P_{3}$, the points in the ball are primarily distributed in one dimension, as in the case of branches. If $P_{1} \approx P_{2} \gg P_{3}$, the points in the ball are primarily distributed in two dimension, as in the case of leaves. Similarly, if $P_{1} \approx P_{2} \approx P_{3}$, the points are distributed evenly in three dimension. Then the salient feature (SF) of the given point at the given scale can be defined as the simple combination of $P_{1}, P_{2}$ and $P_{3}$.

$$
S F=\left(P_{1}, P_{2}, P_{3}\right)
$$

\subsection{Classifier}

The famous machine learning classifier Support Vector Machine (SVM) is used in our method (Vapnik et al.,1997). For a binary classification problem, it tries to find a hyper-plane $\boldsymbol{w} \cdot \boldsymbol{x}+b=0$, which maximizes the distance of the closest vector in both classes. $\boldsymbol{w}$ is the normal vector to the hyper-plane, and $b$ is the distance of the closest point on the hyper-plane to the origin. For a non-linear classification problem, it uses a kernel function implicitly mapping the vector into a high-dimension space to simplify the classification problem.

\subsection{Evaluation}

We use the balanced accuracy $(b a)$ to assess the accuracy of single- and multi-scale classifiers. With $t l, t w, f l, f w$ denotes the number of points $\operatorname{truly}(t) / f a l s e(f)$ classified into the leaf $(l)$ / $\operatorname{wood}(w)$ class, balanced accuracy is classically defined as $b a=$ $(a l+a w) / 2$ with each class accuracy defined as $a l=t l /(t l+$ $f w$ ) and $a w=t w /(t w+f l)$ (Brodu et al., 2012). The total running time of the processing includes the time consumed by feature extracting, classifier training, and leaf and wood classification. The speed-up ratio is used to assess the efficiency of single- and multi-scale classifier. With $t a$ and $t b$ denoting the total running time of classifier $a$ and $b$, speed-up ratio of classifier $a$ over $b$ is defined as $t a / t b$

\section{EXPERIMENTS AND RESULTS}

Our experiments are conducted on a 64-bit windows 10 with an Intel Core $17-7700 \mathrm{k} 4.2 \mathrm{GHz}$ processor and 16GB RAM. The source code of our method is written in $\mathrm{C}++$ programing language. To reduce the computation load, we calculate the salient features on a subset of the tree point cloud. The number of points in the subset is about $10 \%$ of the tree point clouds Firstly, about twenty percent of the aforementioned data is used to train forty single-scale classifiers $(r=0.02 \mathrm{~m}, 0.04 \mathrm{~m}, 0.06 \mathrm{~m}, \ldots$, $0.98 \mathrm{~m}, 1 \mathrm{~m})$ and one multi-scale classifier $(r=0.02 \mathrm{~m}-1 \mathrm{~m})$ for each tree. The reason why we train so many single-scale classifiers is that we want to obtain an optimal average classification accuracy of the single-scale classifier. Then, these classifiers are used to classify leaf and wood points for each tree.

The classified leaf and wood points of tree 1 and tree 2 are shown in Figure 1 and Figure 2, respectively. Owing to the limitation of space, we only plot one single-scale classifier classification result for tree 1 and tree 2 in Figure 1(b) and Figure 2(b),as they has the highest balanced accuracy. Similarly, the classification result of the multi-scale classifier for tree 1 and tree 2 is presented in Figure 1(c) and Figure 2(c), respectively.

The classification accuracies are shown in Figure 3. The highest balanced accuracy of the single-scale classifier $(r=0.3 \mathrm{~m})$ for tree 1 is 0.78 , which is lower than that of the multi-scale classifier by about $4 \%$. The highest balanced accuracy of the single-scale classifier $(r=0.14 \mathrm{~m})$ for tree 2 is 0.82 , which is very close to that of multi-scale classifier. However, the mean balanced accuracy of the single-scale classifiers for each tree is close to 0.7 , which is lower than that of the multi-scale classifiers by about $10 \%$. It is worth noting that the balanced accuracies of each classifier for tree 1 and tree 2 are lower than those reported in Yun et al. (2016), Ma et al. (2016) and Wang et al. (2017) by about $10 \%-15 \%$ because different tree point clouds, salient features and classifiers are used. Because the purpose of our study is to study the abilities of single-scale and multi-scale features to characterize the local spatial patterns of points in 
point clouds, we only build a classifier as simple as Brodu's method.

The speed-up ratios of each single-scale classifier over the multiscale classifier for both trees are shown in Figure 4. The mean speed-up ratios for tree 1 and tree 2 are up to 49.90 and 32.77 , respectively. So more attention should be paid to reducing the computation load if we want to use multi-scale features to improve classification accuracy in the future.

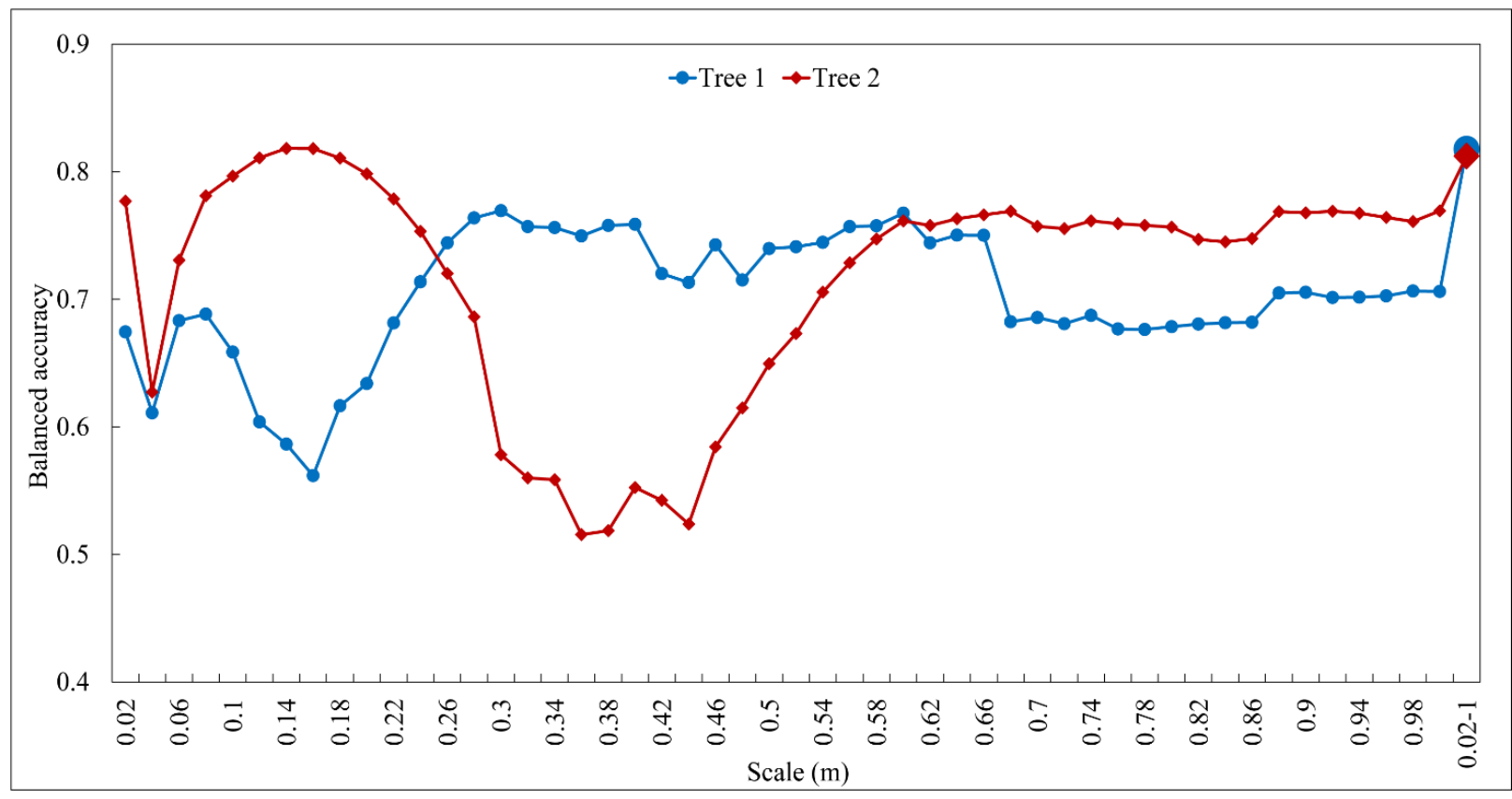

Figure 3. The balanced accuracy of each classifier for each tree. The balanced accuracy of multi-scale classifier is shown in the last column.

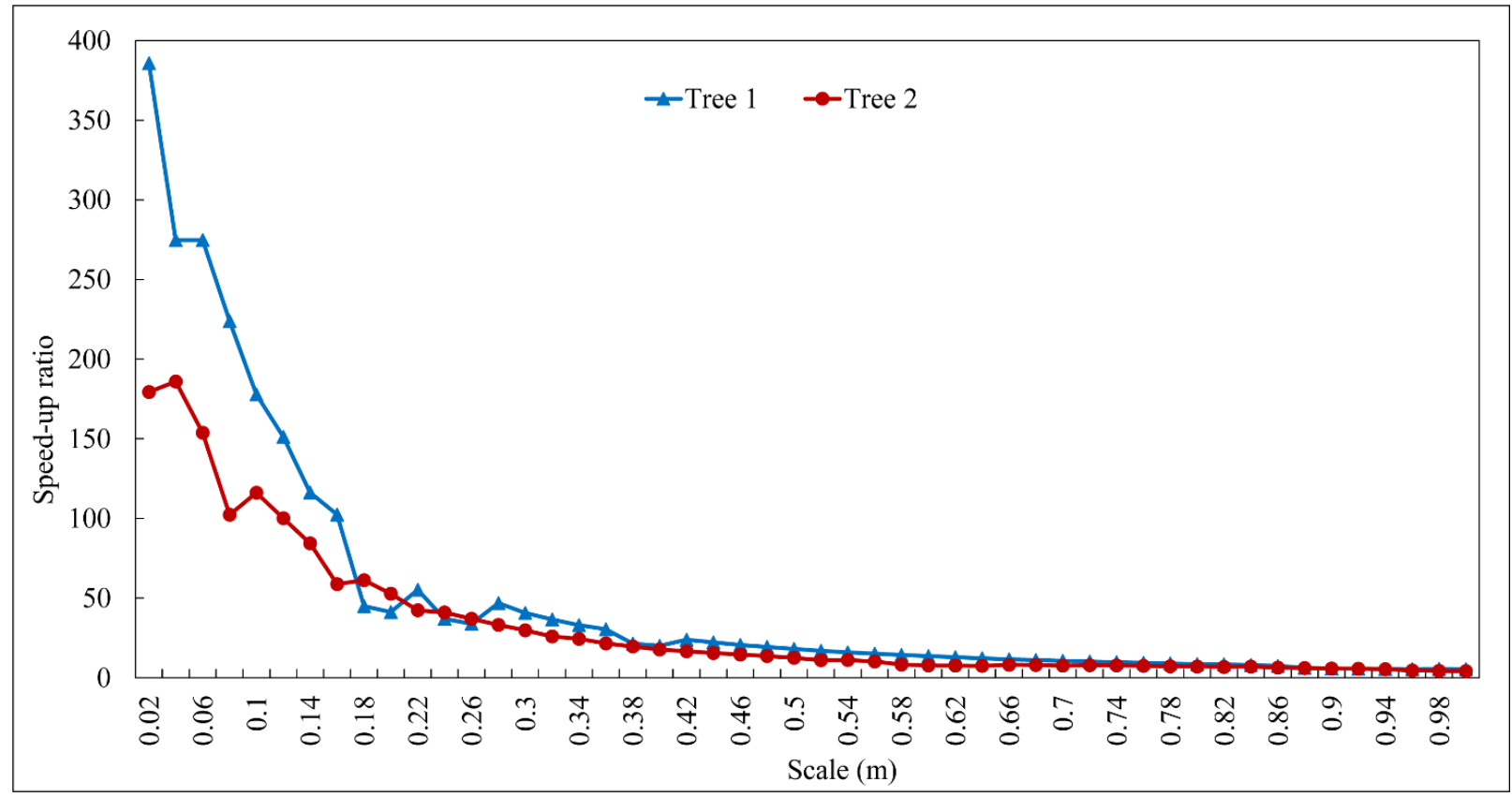

Figure 4. The speed-up ratio of each single-scale classifier over multi-scale classifier for each tree. 


\section{CONCLUSION}

In this study, we assess how single-scale and multi-scale features affect the classification accuracy and efficiency of leaf and wood. Two oak trees of different sizes and complexities are used to evaluate the accuracy and efficiency of the classifiers. Experimental results show that multi-scale features can achieve higher balanced accuracy. On average, the balanced accuracy of the multi-scale method is higher than that of the single-scale method by about $10 \%$. However, the mean speed-up ratio of single scale classifiers over multi-scale classifier is higher than 30. In the future, we will employ more optimization strategies to reduce the processing time of the multi-scale method.

\section{ACKNOWLEDGEMENTS}

This work was supported by the National Natural Science Foundation of China under Grant number 41671427; the Fundamental Research Funds for the Central Universities under Grant number ZYGX2016J148. We thank the referees for their constructive criticism and comments.

\section{REFERENCES}

Béland, M., Baldocchi, D.D., Widlowski, J.L., Fournier, R.A.,and Verstraete, M.M. 2014. On seeing the wood from the leaves and the role of voxel size in determining leaf area distribution of forests with terrestrial LiDAR. Agricultural \& Forest Meteorology, 184(2), pp. 82-97.

Brodu, N., and Lague, D. 2012. 3D terrestrial LiDAR data classification of complex natural scenes using a multi-scale dimensionality criterion: applications in geomorphology. Isprs Journal of Photogrammetry \& Remote Sensing, 68(1), pp.121134.

Calders, K., Disney, M.I., Armston, J., Burt, A., Brede, B., and Origo, N. 2017. Evaluation of the range accuracy and the radiometric calibration of multiple terrestrial LiDAR scanning instruments for data interoperability. IEEE Transactions on Geoscience \& Remote Sensing, 55(5), pp.1-9.

Dassot, M., Constant, T., and Fournier, M. 2011. The use of terrestrial LiDAR technology in forest science: application fields, benefits and challenges. Annals of Forest Science, 68(5), pp.959-
974.

Dey, D., Mummert, L., and Sukthankar, R. 2012. Classification of plant structures from uncalibrated image sequences. In Proceedings of the 2012 IEEE Workshop on Applications of Computer Vision (WACV), Breckenridge, CO, USA, pp.329-336.

Hakala, T., Suomalainen, J., Kaasalainen, S., and Chen, Y. 2012. Full waveform hyperspectral LiDAR for terrestrial laser scanning. Optics Express, 20(7), pp.7119.

Lalonde, J. F., Vandapel, N., Huber, D. F., and Hebert, M. 2006. Natural terrain classification using three-dimensional LiDAR data for ground robot mobility. Journal of Field Robotics, 23(10), pp.839-861.

Li, S., Dai, L., Wang, H., Wang, Y., He, Z., and Lin, S. 2017. Estimating leaf area density of individual trees using the point cloud segmentation of terrestrial LiDAR data and a voxel-based model. Remote Sensing, 9(12), pp.1202.

Ma, L., Zheng, G., Eitel, J.U.H., Moskal, L.M., He,W., and Huang, H. 2016. Improved salient feature-based approach for automatically separating photosynthetic and nonphotosynthetic components within terrestrial LiDAR point cloud data of forest canopies. IEEE Transactions on Geoscience \& Remote Sensing, 54(2), pp.679-696.

Pfeifer, N., Dorninger, P., Haring, A., and Fan, H. 2007. Investigating terrestrial laser scanning intensity data: quality and functional relations. 8th Conference on Optical 3-D Measurement Techniques, Zürich, Switzerland, pp.328

Pfennigbauer, M., and Ullrich, A. 2010. Improving quality of laser scanning data acquisition through calibrated amplitude and pulse deviation measurement. Proc Spie, 7684(1), pp.143-153.

Shaw, P.J.A. 2003. Multivariate statistics for the environmental sciences. London : Arnold.

Tao, S., Guo, Q., Su, Y., Xu, S., Li, Y., and Wu, F. 2015. A geometric method for wood-leaf separation using terrestrial and simulated LiDAR data. Photogrammetric Engineering \& Remote Sensing, 81(10), pp.767-776. 
Vapnik, and Vladimir, N. 1997. The nature of statistical learning theory. IEEE Transactions on Neural Networks, 38(4), pp.409409.

Wang, D., Hollaus, M., and Pfeifer, N. 2017. Feasibility of machine learning methods for separating wood and leaf points from terrestrial laser scanning data. Isprs Annals of the Photogrammetry, IV-2/W4, pp.157-164.

Weinmann, M., Jutzi, B., and Mallet, C. 2013. Feature relevance assessment for the semantic interpretation of 3D point cloud data. Isprs Annals of Photogrammetry, II-5/W2, pp.313-318.

Weinmann, M., Jutzi, B., Hinz, S., and Mallet, C. 2015. Semantic point cloud interpretation based on optimal neighborhoods, relevant features and efficient classifiers. Isprs Journal of Photogrammetry \& Remote Sensing, 105, pp.286-304.

Weinmann, M., Weinmann, M., Mallet, C., and Brédif, M. 2017. A classification-segmentation framework for the detection of individual trees in dense mms point cloud data acquired in urban areas. Remote Sensing, 9(3), pp.277.

Yun, T., An, F., Li, W., Sun, Y., Cao, L., and Xue, L. 2016. A novel approach for retrieving tree leaf area from ground-based LiDAR. Remote Sensing, 8(11), pp.942. 